\title{
Deterministic Entanglement of Photons in Two Superconducting Microwave Resonators
}

\author{
H. Wang, ${ }^{1,2}$ Matteo Mariantoni, ${ }^{1}$ Radoslaw C. Bialczak, ${ }^{1}$ M. Lenander, ${ }^{1}$ Erik Lucero, ${ }^{1}$ M. Neeley, ${ }^{1}$ A. D. O' Connell, ${ }^{1}$ \\ D. Sank, ${ }^{1}$ M. Weides, ${ }^{1}$ J. Wenner, ${ }^{1}$ T. Yamamoto, ${ }^{1,3}$ Y. Yin, ${ }^{1}$ J. Zhao, ${ }^{1}$ John M. Martinis, ${ }^{1}$ and A. N. Cleland ${ }^{1, *}$ \\ ${ }^{1}$ Department of Physics, University of California, Santa Barbara, California 93106, USA \\ ${ }^{2}$ Department of Physics and Zhejiang California International NanoSystems Institute, Zhejiang University, Hangzhou 310027, China \\ ${ }^{3}$ Green Innovation Research Laboratories, NEC Corporation, Tsukuba, Ibaraki 305-8501, Japan
}

(Received 9 November 2010; published 7 February 2011)

\begin{abstract}
Quantum entanglement, one of the defining features of quantum mechanics, has been demonstrated in a variety of nonlinear spinlike systems. Quantum entanglement in linear systems has proven significantly more challenging, as the intrinsic energy level degeneracy associated with linearity makes quantum control more difficult. Here we demonstrate the quantum entanglement of photon states in two independent linear microwave resonators, creating $N$-photon NOON states (entangled states $|N 0\rangle+$ $|0 N\rangle$ ) as a benchmark demonstration. We use a superconducting quantum circuit that includes Josephson qubits to control and measure the two resonators, and we completely characterize the entangled states with bipartite Wigner tomography. These results demonstrate a significant advance in the quantum control of linear resonators in superconducting circuits.
\end{abstract}

DOI: 10.1103/PhysRevLett.106.060401

PACS numbers: 03.65.Yz, 03.67.Lx, 85.25.Cp

Quantum superposition and entanglement have been demonstrated experimentally using spinlike physical systems ranging from atoms to electronic circuits [1-7]. These systems all display strong nonlinearity, and are used because this nonlinearity allows straightforward quantum control by classical means. The quantum control of linear systems, exemplified by the harmonic oscillator, is by contrast more difficult, and has only been achieved using nonlinear intermediaries: Atoms [1,8] to control optical cavities, ions to control ion motion $[9,10]$, and superconducting qubits to control photons in microwave resonators [11-14]. Quantum entanglement of cavity photons still presents a significant challenge: Experiments have demonstrated maximally entangled photons in different polarization modes of the same cavity [15] and in free space [16], but the entanglement of photons in two physically distinct cavities [17-19] has proven more elusive.

Here we show the deterministic generation of entangled photon states in two spatially separated microwave resonators, achieved by manipulating the photon states with a pair of superconducting phase qubits. We use as a benchmark the generation of NOON states [20-24], comprising a total of $N$ photons in the two resonators $(A$ and $B)$, entangled in the quantum state

$$
|\psi\rangle=\frac{1}{\sqrt{2}}\left(|N\rangle_{A}|0\rangle_{B}+|0\rangle_{A}|N\rangle_{B}\right),
$$

with $N$ photons in resonator $A$ and zero in $B$, superposed with the state with the occupation numbers reversed. Such a state has the same degree of entanglement as the Bell state, but with $N$ excitations. We also generate MOON states, in which, e.g., resonator $A$ has $M$ or zero quanta, entangled with resonator $B$ with zero or $N$ quanta. We fully characterize the two-resonator photon states using bipartite
Wigner tomography, which represents a nontrivial extension of single-cavity Wigner tomography $[1,9,12-14]$, and allows us to distinguish entanglement from an incoherent ensemble.

To accomplish this goal, we developed a new quantum circuit comprising two superconducting phase qubits [25] and three microwave resonators. A sketch of the circuit topology is shown in Fig. 1(a). The circuit includes a coupling resonator $C$, connected to both qubits, and two state storage resonators $A$ and $B$, each coupled to one qubit. The resonator frequencies are all different, which allows us to frequency select the qubit-resonator interactions. More detailed information regarding the device design, fabrication and experimental setup can be found elsewhere $[12-14,26]$.

The basic method for generating two-resonator entangled states, illustrated in Fig. 2, is to excite and then entangle the two qubits using the coupling resonator. We can swap the resulting Bell state $|e g\rangle+|g e\rangle$ to the two storage resonators, creating an $N=1 \mathrm{NOON}$ state $|10\rangle+$ $|01\rangle$. If we want to generate higher $N$ photon states, we instead selectively excite each qubit to its next higher energy level $|f\rangle$ [Fig. 1(b)], generating the state $|f g\rangle+$ $|g f\rangle$, thus using the qubits as "qutrits" [27,28]. The required microwave excitation is selective, due to the anharmonicity of the qubits. The qubit excitation is then swapped to each storage resonator through the qubit $|f\rangle \leftrightarrow$ $|e\rangle$ transition, creating a four-fold entangled state $\mid$ eg 10$\rangle+$ $|g e 01\rangle$, where the first two letters indicate the qubit states, and the second two numbers the storage resonator states. We then reexcite the qubits to their $|f\rangle$ states, and again swap the excitation to the resonators, generating $\mid$ eg20 ++ $|g e 02\rangle$. This process can be repeated until the entangled state has $N-1$ photons. In the final step, each qubit's 
(a)
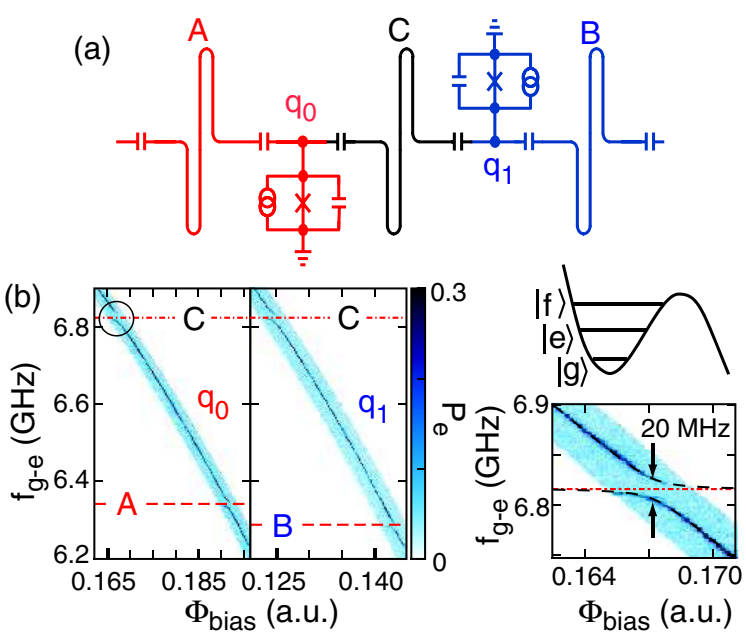

FIG. 1 (color online). (a) Device circuit schematic. Coupling resonator $C$ is connected to $q_{0}$ and $q_{1}$ using $\sim 1.9 \mathrm{fF}$ coupling capacitors that yield $20 \mathrm{MHz}$ coupling strengths, while storage resonator $A(B)$ is connected to $q_{0}\left(q_{1}\right)$ through a $\sim 1.9 \mathrm{fF}$ coupling capacitor with $17 \mathrm{MHz}$ coupling strength. (b) Qubit spectroscopy, showing probability $P_{e}$ (color bar) vs microwave frequency and flux bias for each qubit. Avoided-level crossings near $6.8 \mathrm{GHz}$ (dash-dotted lines) are due to the coupling resonator $C$ and near $6.3 \mathrm{GHz}$ (dashed lines) due to each qubit's storage resonator. Lower right panel shows magnified view of circled area, upper right panel shows three qubit levels.

$|e\rangle \leftrightarrow|g\rangle$ transition is brought on resonance with the corresponding storage resonator, swapping the last excitation and leaving the system in $|g g N 0\rangle+|g g 0 N\rangle=$ $|g g\rangle \otimes(|N 0\rangle+|0 N\rangle)$, an $N$-photon NOON state.

We analyze the final resonator state using the qubits as probes. The simplest analysis uses a coincidence measurement: We bring the qubits into resonance with their corresponding storage resonators for an interaction time $\tau$, after which both qubits are measured simultaneously. The preparation and measurement sequence is repeated $\sim 10^{3}$ times, yielding the joint-qubit state probabilities, $P_{g g}, P_{g e}$, $P_{e g}$, and $P_{e e}$, where $P_{g e}$ is the probability of measuring the first qubit in its ground state with the second qubit in its excited state, and so on. We then vary the interaction time $\tau$, capturing the evolution of these probabilities. If a resonator has $n$ photons, the $n$th photon will swap between the qubit and resonator at a rate scaling as $\sqrt{n}$, while for more complicated states, the interaction is a sum of components oscillating at their respective frequencies, weighted by the photon occupation probabilities $[13,14]$.

For resonators entangled in a NOON state, a joint measurement should correspond to either $N$ photons in one resonator and zero in the other, or to the reverse situation; the measurement of the qubits "collapses" the system onto one or the other outcome. Thus in one measurement at most one of the qubits will be in the excited state. When averaged over many measurements, the maximum probability of measuring a particular qubit in $|e\rangle$ is $1 / 2$,
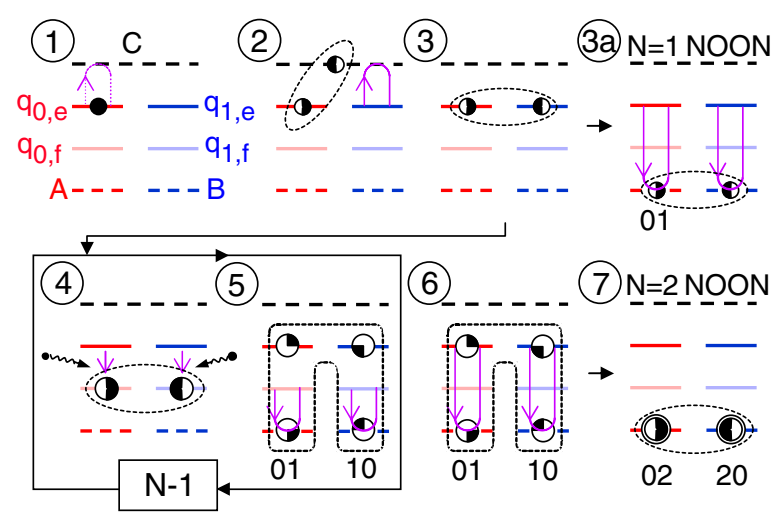

FIG. 2 (color online). NOON state preparation sequence. Resonators are represented by dashed lines and the qubit $|g\rangle \leftrightarrow$ $|e\rangle$ and $|e\rangle \leftrightarrow|f\rangle$ transitions by dark and light (color) solid lines, respectively. (1) $q_{0}$ is excited to $|e\rangle$ and half-swapped to $C$, generating the Bell state $|e 0\rangle+|g 1\rangle$. (2) Coupling resonator swapped to $q_{1}$, generating $|e g\rangle+|g e\rangle$. (3a) $N=1$ NOON state $|10\rangle+|01\rangle$ generated by fully swapping each qubit to its storage resonator. For higher $N$ states: (4) Qubits excited to $|f g\rangle+|g f\rangle$. (5) One photon swapped into storage resonators, generating $|e g 10\rangle+|g e 01\rangle$. Steps (4) and (5) are repeated $N-1$ times, generating $|e g(N-1) 0\rangle+|g e 0(N-1)\rangle$. (6) Final photon transfer generates (7) $N=2$ (or higher $N$ ) NOON state.

while the probability of measuring both qubits in $|e\rangle$ should be zero. Therefore we expect that $P_{e g}$ and $P_{g e}$ will oscillate between 0 and $1 / 2, P_{e e}$ will be zero, and $P_{g g}$ should equal $1-P_{g e}-P_{e g}-P_{e e}$.

Coincidence measurements are shown in Fig. 3 for NOON states up to $N=3$, and are consistent with these expectations. The oscillations seen in these measurements are, however, insufficient proof of resonator entanglement, as an incoherent mixed state can give the same results. To demonstrate this, we have controllably generated a

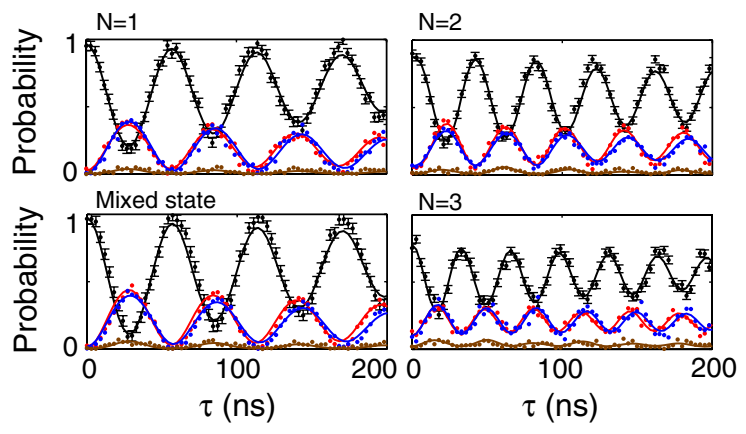

FIG. 3 (color online). Qubit coincidence probability measurements for $N=1,2$, and 3 NOON states, and for a mixed state. $P_{g e}$ (blue) and $P_{e g}$ (red) oscillate with interaction time $\tau$ at a rate $\propto \sqrt{N}$, while $P_{e e}$ (brown) always remains small. For the mixed state, behavior is nearly identical to the $N=1 \mathrm{NOON}$ state. Lines are fits to the data. Statistical errors, from the measured probability spread of $\sim 2 \%-3 \%$, are shown only for $P_{g g}$ [26]. Horizontal and vertical axes are same for all plots. 
synthetic ensemble comprising a $50 \%$ population of $|10\rangle$ states and 50\% $|01\rangle$ states [26]. Coincidence measurements [Fig. 3] of this synthetic mixed state generate outcomes identical to those of the $N=1 \mathrm{NOON}$ state.

A more complete resonator measurement, that can resolve entangled from mixed states, uses bipartite Wigner tomography, a significant extension of single resonator tomography $[9,13,14]$. This involves injecting a coherent Gaussian microwave pulse into each of the storage resonators, with controlled amplitude and phase, displacing the resonator states in phase space. The resonators are then simultaneously measured with a joint probability measurement, now as a function of the amplitude and phase of the coherent pulses. From the complete set of measurements, the two-resonator density matrix can be calculated [26].

In Fig. 4 we display the amplitudes of the density matrices measured for resonator NOON states up to $N=3$, as well as for the mixed state. While there are nonidealities, the desired nonzero matrix elements are clearly apparent for the NOON states, while for the mixed state, the density matrix has only zero-valued off-diagonal elements. The state preparation fidelities, $F=\langle\psi|\rho| \psi\rangle$, are found to be $0.76 \pm 0.02(N=1), 0.50 \pm 0.02(N=2)$, and $0.33 \pm 0.02(N=3)$. For $N=1$, the most probable entanglement of formation (EOF) [29] is $\mathrm{EOF}=0.51$, while for $N=2$ and $N=3$, $\mathrm{EOF}=0.31$ and 0.28 , respectively; for the mixed state, the EOF is zero. We also calculate the negativity $N_{e}(\rho)=\sum_{j} \max \left(0, \mu_{j}\right)$, where $\mu_{j}$ are the eigenvalues of the partial transpose $\rho^{\mathrm{PT}}$ of the density matrix [22,30], and $N_{e}(\rho)>0$ indicates entanglement. The negativities are found to be $0.56 \pm 0.03$ $(N=1), 0.32 \pm 0.03(N=2)$, and $0.27 \pm 0.01(N=3)$; for the mixed state we find zero with an upper bound of 0.001 . The decrease of these values with photon number
$N$ is compatible with expectations: The state preparation requires phase coherence of the four-element entangled states for most of the preparation sequence, which is limited by the qubit coherence time $T_{2}$ [26]. Other than this technical limitation, the deterministic generation is completely scalable to large $N$.

A hallmark of NOON states is their rapid phase evolution $[20,21,23,24]$, which can be verified by Wigner tomography using two distinct methods. For the $N=1$ state, after entangling the qubits in a Bell state, we wait for a variable time and then swap the state into the storage resonators. The density matrices measured at three different delay times are shown in Fig. 5(a). The phases of the off-diagonal elements rotate with time due to the qubitresonator frequency difference, as in Fig. 5(b), showing the expected linear dependence.

This phase-measurement method suffers from the short qubit dephasing time. A second method is to change the phase reference for the coherent pulses used in the Wigner tomography, avoiding storage of the state in the qubit. We add an additional phase to the pulses applied to resonator $A$ only. The resulting density matrices show the expected rotation of the off-diagonal elements. In Fig. 5(c) we plot the off-diagonal phase angle for different $N$; the $N=3$ state evolves 3 times faster than the $N=1$ state, as expected.

We also used tomography to measure the NOON state decay [26]. We find that the off-diagonal elements decrease at approximately the same rate as the diagonal elements, with a decay time $\tau_{D} \approx 3 \mu \mathrm{s}$, consistent with environmental fluctuations that are uncorrelated in time [14] and to a limited degree in space, the latter supported by the absence of correlation between the two resonators. In essence, we have performed a two-point sample of the noise correlation in space.

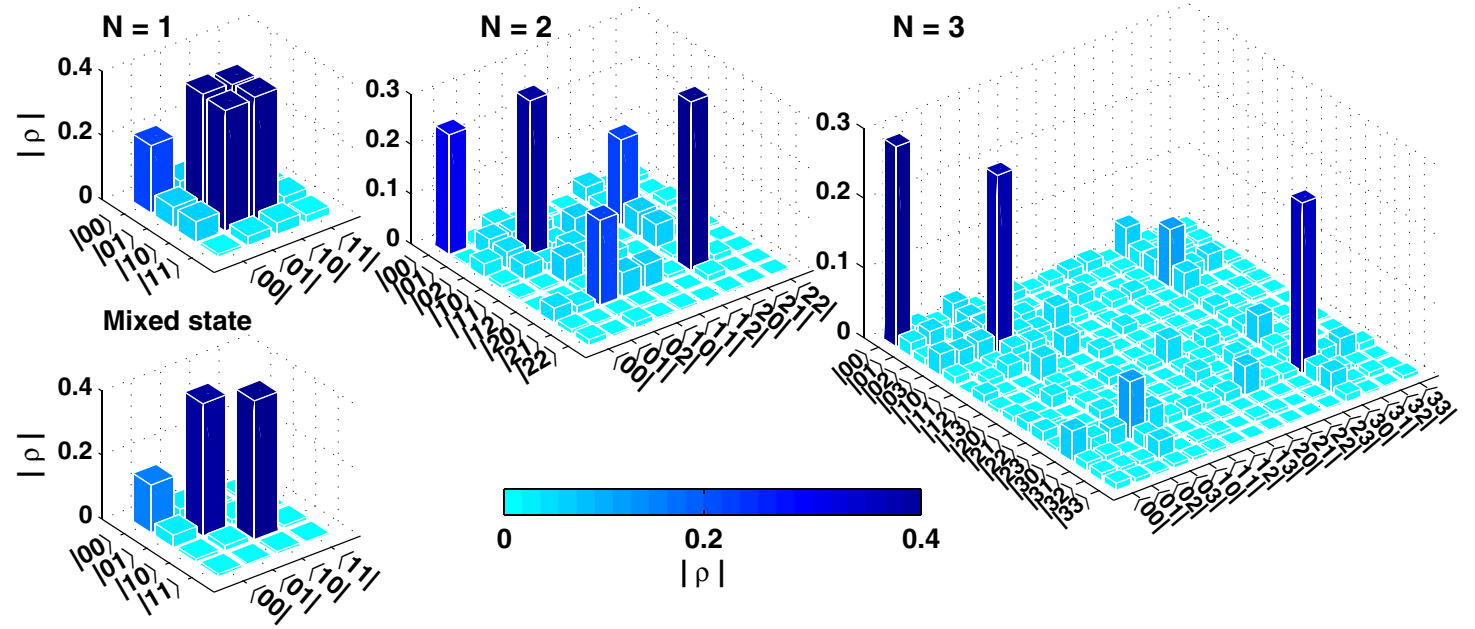

FIG. 4 (color online). NOON and mixed state density matrix amplitudes, reconstructed in the photon-number basis from bipartite Wigner tomography; states are labeled $|m n\rangle$ where $m$ is the photon number in resonator $A$ and $n$ that in $B$. Bar heights and colors represent matrix element amplitudes. The dominant amplitudes for all three NOON states are in the expected locations, although the off-diagonal elements decrease with $N$, due to the finite qubit dephasing time. Errors for the density matrix elements are not shown but are small [26]. 

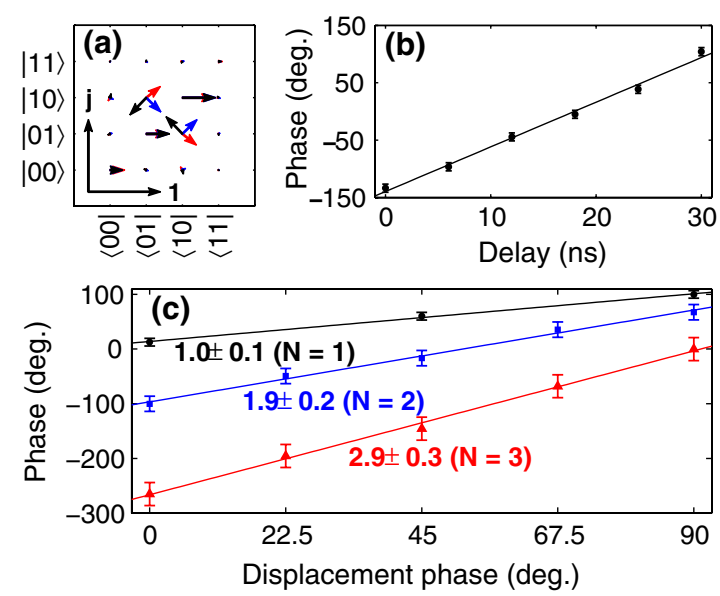

FIG. 5 (color online). Phase sensitivity of NOON states up to $N=3$. (a) Density matrix for $N=1 \mathrm{NOON}$ state at different times after qubit entanglement. Each element is represented by an arrow, with orientation determined by the phase angle (scale on bottom left). Off-diagonal elements rotate with delay time (black: $0 \mathrm{~ns}$, blue: $12 \mathrm{~ns}$, red: $24 \mathrm{~ns}$ ), due to frequency difference between the qubit operating point and resonator. Dephasing causes a decrease in amplitude of off-diagonal elements. (b) Phase angle of the upper left nonzero off-diagonal element in (a) versus delay time. Line is fit to a rotation rate of $\Delta f=21.6 \pm 0.8 \mathrm{MHz}$, which corresponds well to set frequency difference. Error bars indicate maximum phase uncertainty. (c) Rotation angle of off-diagonal element versus controlled, additional phase angle used in coherent state displacement. Lines are fits (slopes indicated on plot), consistent with expected phase sensitivity. Confidence bounds are as in (b), with uncertainty increasing with $N$ due to increased phase sensitivity.

We note that the generation sequence allows an additional flexibility: We can add different numbers of quanta to the resonators, thus generating MOON states, $|\psi\rangle=$ $|M 0\rangle+|0 N\rangle$. An example with $M=2$ and $N=1$ is detailed in [26].

The capabilities we have demonstrated here, generating complex entangled photon states in two resonators, hold promise for new quantum architectures in which superconducting resonators play a more central role. The protocol can be extended in a natural way to entangle larger numbers of resonators, allowing, e.g., the direct generation of resonator Greenberger-Horne-Zeilinger and W states $[2,4,9,16,21,23]$. The longer coherence times achievable in superconducting resonators will be of direct utility in performing more complex quantum algorithms, furthering the capabilities of superconducting quantum circuit architectures.

We thank K. Audenaert for assistance in entanglementof-formation calculations. This work was supported by IARPA under ARO Grant No. W911NF-04-1-0204. M.M. acknowledges support from the Elings
Postdoctoral Fellowship program. H.W. acknowledges partial support by the Fundamental Research Funds for the Central Universities in China (Program No. 2010QNA3036). Devices were made at the UC Santa Barbara Nanofabrication Facility, a part of the NSF-funded National Nanotechnology Infrastructure Network.

*Corresponding author. cleland@physics.ucsb.edu

[1] S. Haroche and J. M. Raimond, Exploring the QuantumAtoms, Cavities and Photons (Oxford University Press, Oxford UK, 2006).

[2] R. Blatt and D. Wineland, Nature (London) 453, 1008 (2008).

[3] R. Hanson, L. P. Kouwenhoven, J. R. Petta, S. Tarucha, and L. M. K. Vandersypen, Rev. Mod. Phys. 79, 1217 (2007).

[4] P. Neumann et al., Science 320, 1326 (2008).

[5] J. Clarke and F. K. Wilhelm, Nature (London) 453, 1031 (2008).

[6] M. Neeley et al., Nature (London) 467, 570 (2010).

[7] L. DiCarlo et al., Nature (London) 467, 574 (2010).

[8] H. Mabuchi and A.C. Doherty, Science 298, 1372 (2002).

[9] D. Leibfried, R. Blatt, C. Monroe, and D. J. Wineland, Rev. Mod. Phys. 75, 281 (2003).

[10] J. D. Jost et al., Nature (London) 459, 683 (2009).

[11] R. J. Schoelkopf and S. M. Girvin, Nature (London) 451, 664 (2008).

[12] M. Hofheinz et al., Nature (London) 454, 310 (2008).

[13] M. Hofheinz et al., Nature (London) 459, 546 (2009).

[14] H. Wang et al., Phys. Rev. Lett. 103, 200404 (2009).

[15] A. Rauschenbeutel et al., Phys. Rev. A 64, 050301(R) (2001).

[16] S. B. Papp et al., Science 324, 764 (2009).

[17] M. Mariantoni et al., Phys. Rev. B 78, 104508 (2008).

[18] S. T. Merkel and F. W. Wilhelm, New J. Phys. 12, 093036 (2010).

[19] F. W. Strauch, K. Jacobs, and R. W. Simmonds, Phys. Rev. Lett. 105, 050501 (2010).

[20] A. N. Boto et al., Phys. Rev. Lett. 85, 2733 (2000).

[21] T. Nagata et al., Science 316, 726 (2007).

[22] L. K. Shalm, R.B.A. Adamson, and A. M. Steinberg, Nature (London) 457, 67 (2009).

[23] J. A. Jones et al., Science 324, 1166 (2009).

[24] I. Afek, O. Ambar, and Y. Silberberg, Science 328, 879 (2010).

[25] J. M. Martinis, Quant. Info. Proc. 8, 81 (2009).

[26] See supplementary material at http://link.aps.org/ supplemental/10.1103/PhysRevLett.106.060401.

[27] J. Q. You, Y.-X. Liu, C. P. Sun, and F. Nori, Phys. Rev. B 75, 104516 (2007).

[28] M. Neeley et al., Science 325, 722 (2009).

[29] K. Audenaert, F. Verstraete, and B. DeMoor, Phys. Rev. A 64, 052304 (2001).

[30] A. Peres, Phys. Rev. Lett. 77, 1413 (1996). 